\title{
All-Optical Multicast Switch Employing Raman-Assisted FWM in Dispersion-Shifted Fiber
}

\author{
Kwan Lau, Member, IEEE, S. H. Wang, Lixin Xu, C. Lu, Member, IEEE, H. Y. Tam, Senior Member, IEEE,
} and P. K. A. Wai, Senior Member, IEEE

\begin{abstract}
We demonstrate an all-optical multicast switch with the capability to select five reconfigurable channel groups with six output multicast channels using three control wavelengths at $10 \mathrm{~Gb} / \mathrm{s}$ using Raman-assisted four-wave mixing in dispersion-shifted fiber. All the output channels comply with the $100 \mathrm{GHz}$ (i.e., $0.8 \mathrm{~nm}$ ) spaced International Telecommunication Union (ITU) grid.
\end{abstract}

Index Terms-All-optical devices, nonlinear optics, optical communication, optical multicast.

\section{INTRODUCTION}

$\mathbf{O}$ PTICAL multicast is an important and exciting research area [1] because many future broadband applications such as high definition Internet TV can be realized without the need for O-E-O conversion at the lower layers. Optical multicast on data plane was proposed and demonstrated using various techniques [2]-[6]. However, the proposed multicast schemes so far are designed for fixed multicast and the effort has mainly been on increasing the number of multicast channels [4], [5]. They are not reconfigurable and inefficient to change the multicast group members with control plane information.

In an Internet Protocol (IP) network such as the Internet, group membership protocol and multicast routing protocol are used to support IP multicasting across the IP network. This allows the multicast capable routers to send data only to the hosts that belong to the group. In order to map the control information of these protocols to the data plane lightpath connections, optical multicast switches need to be reconfigurable based on the control plane information. The possibility was indirectly demonstrated for three controllable multicast channels in [6] where every channel requires a control wavelength. No controllable optical multipath switch with more than three channels, however, has been demonstrated up to now. To the best of our knowledge, we are the first to demonstrate experimentally an all-optical multicast switch capable of selecting a mul-

Manuscript received April 27, 2008; revised August 5, 2008. Current version published September 26, 2008. This work was supported in part by The Hong Kong Polytechnic University under Project 1-BBZB.

K. Lau, S. H. Wang, C. Lu, and P. K. A. Wai are with the Department of Electronic and Information Engineering, The Hong Kong Polytechnic University, Kowloon, Hong Kong, China (e-mail: kwan.lau@polyu.edu.hk; kwanchi.lau@motorola.com; 05900629r@polyu.edu.hk; enluchao@polyu. edu.hk; enwai@inet.polyu.edu.hk).

L. Xu is with the Department of Physics, University of Science and Technology of China, Hefei 230026, China (e-mail: xulixin@ustc.edu.cn).

H. Y. Tam is with the Department of Electrical Engineering, The Hong Kong Polytechnic University, Kowloon, Hong Kong, China (e-mail: eehytam@ polyu. edu.hk).

Digital Object Identifier 10.1109/LPT.2008.2004565

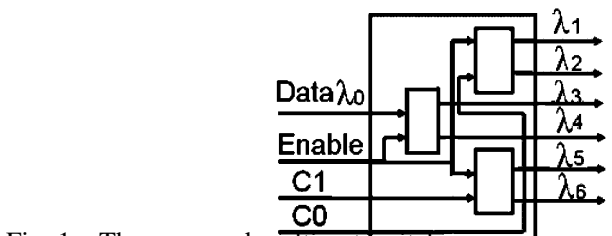

Fig. 1. The proposed multicast twitch

TABLE I

InPUT/OutPut TABLE OF Multicast SWITCH WHERE $1=$ WAVELENGTH IS PRESENT, $0=$ No WAVELENGTH IS PRESENT, AND $\mathrm{X}=$ DON'T CARE

\begin{tabular}{|l|l|l|l|l|l|l|l|l|}
\hline Input & \multicolumn{6}{|c|}{ Output } \\
\hline Enable & $\mathbf{C 1}$ & $\mathbf{C} 0$ & $\boldsymbol{\lambda}_{\mathbf{1}}$ & $\boldsymbol{\lambda}_{\mathbf{2}}$ & $\boldsymbol{\lambda}_{\mathbf{3}}$ & $\boldsymbol{\lambda}_{\mathbf{4}}$ & $\boldsymbol{\lambda}_{\mathbf{5}}$ & $\boldsymbol{\lambda}_{\mathbf{6}}$ \\
\hline 0 & $\mathrm{X}$ & $\mathrm{X}$ & 0 & 0 & 0 & 0 & 0 & 0 \\
\hline 1 & 0 & 0 & 0 & 0 & 1 & 1 & 0 & 0 \\
\hline 1 & 1 & 0 & 0 & 0 & 1 & 1 & 1 & 1 \\
\hline 1 & 0 & 1 & 1 & 1 & 1 & 1 & 0 & 0 \\
\hline 1 & 1 & 1 & 1 & 1 & 1 & 1 & 1 & 1 \\
\hline
\end{tabular}

ticast channel group in the data plane based on control plane information.

In this letter, we propose to use Raman-assisted four-wave mixing (FWM) because of its high efficiency to demonstrate an all-optical multicast switch. A multicast switch with six output channels was demonstrated experimentally with the capability to select five reconfigurable channel groups using three control wavelengths.

\section{OPERATING PRINCIPLE}

Fig. 1 shows the proposed multicast switch which consists of three $1 \times 2$ switches as the basic building block of the switch. The $1 \times 2$ switch can be easily implemented through the use of FWM to generate two copies of the same signal. However, the scaling up of the switch is limited by the low FWM efficiency. This problem has been solved through the use of Raman-assisted FWM in a dispersion-shifted fiber (DSF) and the proposed switch has been implemented by cascading three $1 \times 2$ switches. The three control wavelengths (Enable, C0, and $\mathrm{C} 1$ ) are used to select the multicast channels to provide support for multicast group membership protocol such as the Internet group membership protocol (IGMP) which is widely used in the IP multicast network. By using the header processing technique of the all-optical packet switch proposed in [7], it is possible to demonstrate an all-optical reconfigurable multicast packet switch which is capable of selecting multicast channel groups by the header information in a packet. Table I shows the input/ output $(\mathrm{I} / \mathrm{O})$ relationships of the multicast switch where Enable is the master control. The state of $\mathrm{C} 0$ and $\mathrm{C} 1$ will matter only when the master control is in "Enable" state. Although it 
is possible to have eight combinations of channel groups with three control signals, the implementation will be complicated and may not even be possible if FWM is used alone.

The idea of using Raman-assisted FWM has been proposed for its high efficiency [8], which is highly desirable for optical multicast systems containing multiple channels. FWM also offers transparency in both bit rate and modulation format. In other multicast schemes employing FWM in DSF without Raman assistance, high CW pump powers are required to achieve high FWM efficiency [6]. Therefore, polarization beam splitters (PBS) are commonly used to generate orthogonal pumps and channels to minimize the beatings and interaction of pumps in order to maximize the FWM efficiency. In our proposed scheme no PBS is used since efficiency is mainly improved through the Raman pump. Since backward pumped Raman gain has low polarization dependence, it helps to reduce the beatings among pumps and thus simplifies the setup. However, polarization controllers (PC) are still required since FWM is polarization sensitive. The operating wavelengths are selected at $1550-60 \mathrm{~nm}$, where the maximum efficiency is given. It is due to the Raman pump wavelength of $1455 \mathrm{~nm}$ which has the peak gain at around $1555 \mathrm{~nm}$. Phase matching is an important condition for a FWM process. Therefore, the operating wavelength should be near the zero dispersion wavelength (ZDW) of the DSF in order to achieve the phase matching to maximize the FWM efficiency which would also increase the beatings among pumps. Using range of $1550 \mathrm{~nm}$ instead of the ZDW $(\sim 1538 \mathrm{~nm})$ is also useful to reduce the beatings by limiting the phase matching region with relative large dispersion.

We use three continuous wave $(\mathrm{CW})$ pumps to work as multicast controls (Enable, $\mathrm{C} 0$, and $\mathrm{C} 1$ ). The wavelengths of data $\left(\lambda_{\mathrm{d}}\right)$ and Enable $\left(\lambda_{\mathrm{e}}\right)$ are used to start the FWM process which generates channel $3\left(\lambda_{3}\right)$ and $4\left(\lambda_{4}\right)$. The formula of channel frequencies in a degenerated FWM process is: $f_{\mathrm{d}}+f_{\mathrm{e}}=f_{3}+f_{4}$. The selection of control wavelengths (i.e., Enable, C0, and C1) is based on the configuration shown in Fig. 1 such that it provides the best results. Both $\mathrm{C} 0$ and $\mathrm{C} 1$ are selected to be between a pair of multicast channels (i.e., 1 and 2 , and 5 and 6 ) with channel spacing of $0.8 \mathrm{~nm}$ to comply with $100-\mathrm{GHz}$ ITU grid.

We notice that all six channels are generated as a result of FWM even when the Raman pump is switched off (Fig. 2). However, the power is too low $(<-50 \mathrm{dBm})$. The Raman pump in our scheme is used mainly as an amplifier to improve the efficiency of generating the multicast wavelength channels. In a FWM process, the output power of FWM light is proportional to the power of the three input lights. In Raman-assisted FWM, stimulated Raman scattering (SRS) offers overall gain to the FWM process and also contributes a nonlinear phase shift to the phase-matching condition [9]. As the channels and pumps are amplified by Raman pump, the increases in power of CW pumps and channels change the output of the FWM processes owing to SRS. Fig. 2 shows that both channels 2 and 5 have less power than channels 1 and 6 when the Raman pump is off. When the Raman pump is on, both channels 2 and 5 have larger power than 1 and 6 . It demonstrates how the Raman pump "assists" the FWM in the system. Since wavelengths of data and

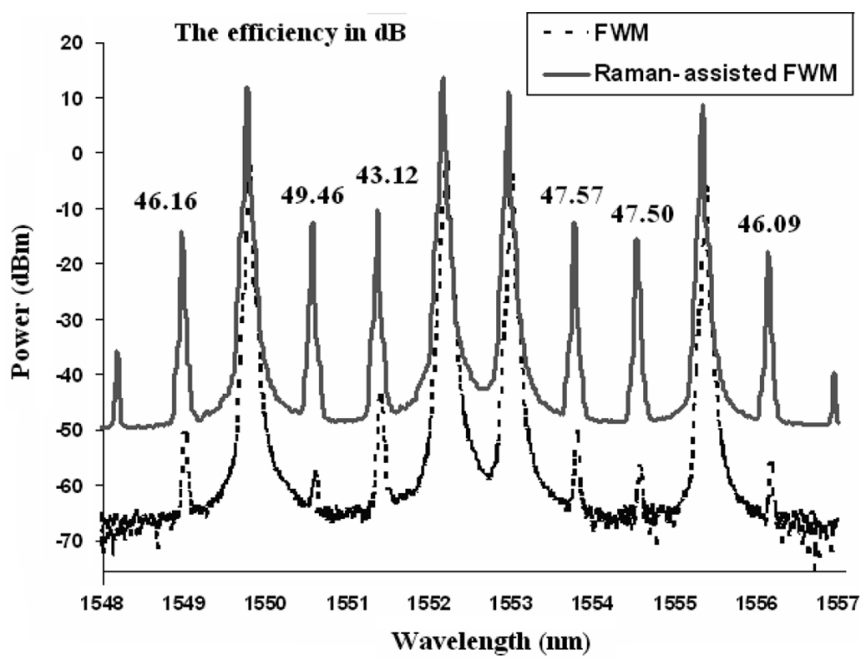

Fig. 2. Output optical spectra with and without Raman assistance. The number is the efficiency gain in decibels for each multicast channel.

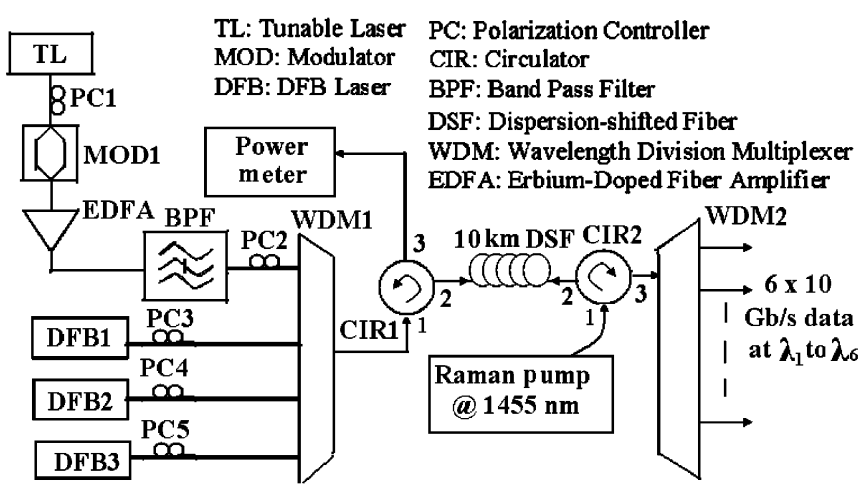

Fig. 3. Experimental setup.

"Enable" start the first FWM process, nothing will happen if Enable wavelength is not present in the system in the first place. When Enable wavelength is present, channels 1 and 2 will not be available if $\mathrm{C} 0$ wavelength is not present. Similarly, channels 5 and 6 will not be available in the absence of C1. By making use of such observations, we are able to select the five wavelength groups by the three control wavelength and achieve what is shown in Table I.

\section{EXPERIMENTAL RESULTS}

Fig. 3 shows the experimental setup. The $10-\mathrm{Gb} / \mathrm{s}$ nonreturn-to-zero (NRZ) data signal at $1552.2 \mathrm{~nm}\left(\lambda_{\mathrm{d}}\right)$ was generated by external modulation of a tunable laser with the power of $5.13 \mathrm{dBm}$. The wavelengths and powers of the CW pumps generated by DFB lasers are $1549.8 \mathrm{~nm}\left(\lambda_{\mathrm{CO}}\right)$ and $4.25 \mathrm{dBm}$ for $\mathrm{C} 0,1553 \mathrm{~nm}\left(\lambda_{\mathrm{e}}\right)$ and $2.63 \mathrm{dBm}$ for Enable, and $1555.4 \mathrm{~nm}\left(\lambda_{\mathrm{C} 1}\right)$ and $0.36 \mathrm{dBm}$ for $\mathrm{C} 1$, respectively. The measurements are taken at port 1 of the circulator 1 (CIR1). The wavelength separation between data signal and the $\mathrm{CW}$ pumps are multiples of $0.8 \mathrm{~nm}$. The data signal and the pumps were combined with a wavelength division multiplexer (WDM1) after passing through the polarization controllers PC2, PC3, PC4, and PC5, respectively. The combined signals were then launched into a $10-\mathrm{km}$ DSF, which has the zero-dispersion wavelength at $\sim 1538 \mathrm{~nm}$, through a circulator (CIR1). The 

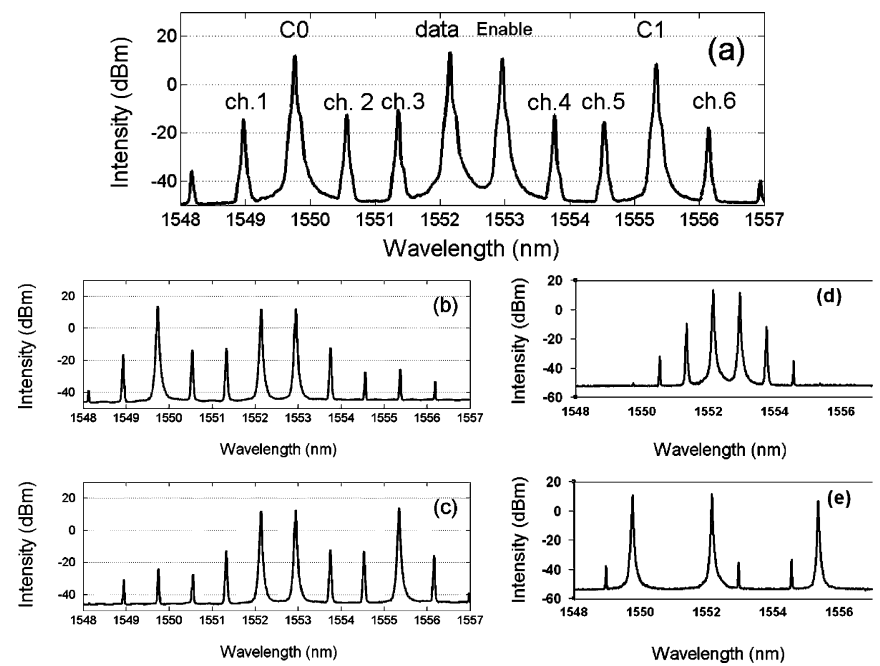

Fig. 4. Output spectrum when (a) all three pumps $(\mathrm{C} 0, \mathrm{C} 1$, and Enable) are present, (b) $\mathrm{C} 0$ and Enable pumps are present, (c) $\mathrm{C} 1$ and Enable pumps are present, (d) only Enable is present, and (e) only Enable is not present.

nonlinear coefficient of the DSF is $\gamma=2 \mathrm{~W}^{-1} \cdot \mathrm{km}^{-1}$ and the fiber attenuation at $1550 \mathrm{~nm}$ is $0.2 \mathrm{~dB} / \mathrm{km}$. The Raman pump at $1455 \mathrm{~nm}$ is coupled into the DSF through another circulator (CIR2) in the counterpropagating direction. The Raman pump power at the input of the DSF is $1.4 \mathrm{~W}$, which is used to compensate the low FWM efficiency and provide the gain required for newly generated channels. The newly generated signals were obtained from the output ports of WDM2 and sent to the optical spectrum analyzer and the $50 \mathrm{GHz}$ oscilloscope for spectrum and time domain analysis, respectively. A variable optical attenuator (VOA) controls the received optical power at a $10-\mathrm{Gb} / \mathrm{s}$ bandwidth PIN photodiode for bit-error rate (BER) measurement.

Fig. 4(a)-(e) shows the output spectrum of the channels for five cases: (a) all three pumps are present; (b) Enable and C0 pumps are present; (c) Enable and $\mathrm{C} 1$ pumps are present; (d) only Enable is present; and (e) only Enable is not present. The results agree with what is given in Table I and also demonstrate how multicast groups are selected optically. In all cases, the power of all multicast channels which have been generated, respectively, through the use of Raman-assisted FWM was larger than $-20 \mathrm{dBm}$. The power of all unwanted channels $(<-30 \mathrm{dBm})$ is at least $10 \mathrm{~dB}$ below that of the signal channels.

Fig. 2 shows the output spectrum of the multicast switch with and without Raman assistance where every wavelength enjoys a 40 to $50 \mathrm{~dB}$ gain. No significant change in power (less than $2 \mathrm{~dB}$ ) and eyes of individual channels were observed by switching $\mathrm{C} 0$ and $\mathrm{C} 1$ on and off, respectively. The performance of the system in case $3 \mathrm{a}$ was investigated by measuring the BER performance of 10-Gb/s signals in all six channels, which is shown in Fig. 5. We noted that the output signal performances of the channels were similar. The average power penalty was $\sim 2.0 \mathrm{~dB}$ at BER of $10^{-9}$ and the largest power penalty was $\sim 2.3 \mathrm{~dB}$ which was observed at channel 6 .

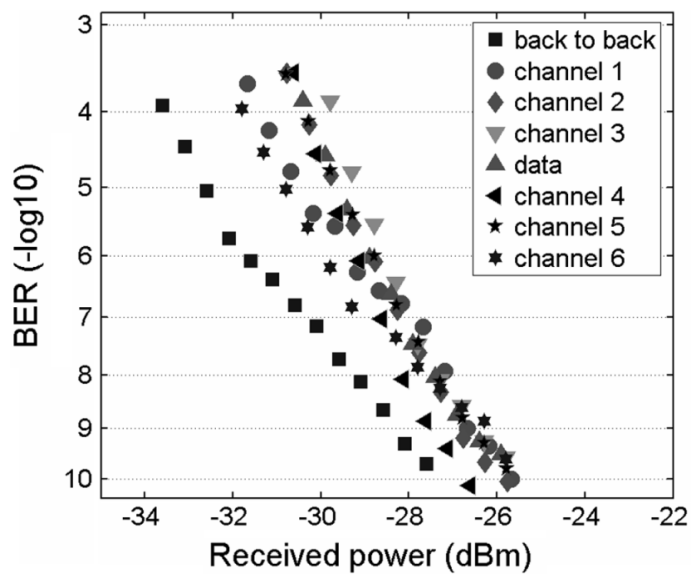

Fig. 5. BER of multicast wavelength channels.

\section{CONCLUSION}

We proposed and demonstrated experimentally an all-optical multicast switch employing Raman-assisted FWM in DSF with wavelength control capability to select the multicast channel groups. All six channels $(10 \mathrm{~Gb} / \mathrm{s}$ per channel) were obtained with an average power penalty of $\sim 2 \mathrm{~dB}$ at BER of $10^{-9}$. There is no quality degradation on channels from multicast group switching. All the output channels comply with $10 \mathrm{GHz}$ (i.e., $0.8 \mathrm{~nm}$ ) spaced ITU grid. High efficiency gain of around $50 \mathrm{~dB}$ was achieved on every channel. The inherent high speed nature of FWM should allow the proposed multicast scheme to operate at bit rates higher than $10 \mathrm{~Gb} / \mathrm{s}$ demonstrated here. Further increase on the number of controllable multicast channels can be realized through the use of highly nonlinear fiber and the increase of the number of control wavelengths.

\section{REFERENCES}

[1] J. George and N. Rouskas, "Optical layer multicast: Rationale, building blocks, and challenges," IEEE Network, vol. 17, no. 1, pp. 60-65, Jan./ Feb. 2003.

[2] W. S. Hu and Q. J. Zeng, "Multicasting optical cross conects employing splitter-and-delivery switch," IEEE Photon. Technol. Lett., vol. 10, no. 7, pp. 970-972, Jul. 1998.

[3] J. L. Pleumeekers et al., "All-optical wavelength conversion and broadcasting to eight separate channels by a single semiconductor optical amplifier delay interferometer," in Proc. OFC, 2002, pp. 596-597.

[4] Q. Lin, R. Jiang, C. F. Marki, C. J. McKinstrie, R. Jopson, J. Ford, G. P. Agrawal, and S. Radic, " $40 \mathrm{~Gb} / \mathrm{s}$ optical switching and wavelength multicasting in a two pump parametric device," IEEE Photon. Technol. Lett., vol. 10, no. 11, pp. 2376-2378, Nov. 2005.

[5] C. S. Bres et al., "1-to-40 Multicasting and amplification of $40 \mathrm{Gbps}$ channels in wideband parametric amplifier," in Proc. OFC/NFOEC 2008, San Diego, CA, Paper PDP16.S.

[6] Y. Wang, C. Yu, T. Luo, L. Yan, Z. Pan, and A. E. Willner, "Tunable all-optical wavelength multicasting using orthogonally-polarized fiber FWM," J. Lightw. Technol., vol. 23, no. 10, pp. 3331-3338, Oct. 2005.

[7] P. K. A. Wai, L. Y. Chan, L. F. K. Lui, L. Xu, H. Y. Tam, and M. S. Demokan, " $1 \times 4$ all-optical packet switch at $10 \mathrm{~Gb} / \mathrm{s}$," IEEE Photon. Technol. Lett., vol. 17, no. 6, pp. 1289-1291, Jun. 2005.

[8] L. Xu, P. K. A. Wai, and H. Y. Tam, "Raman-assisted four-wave mixing," in Proc. 6th Chinese OptoElectronics Symp. (COES'2003), 2003, pp. 169-171.

[9] S. H. Wang, L. Xu, P. K. A. Wai, and H. Y. Tam, "Wavelength conversion using multi-pump Raman-assisted four-wave mixing," in Proc. CLEO 2007, Baltimore, MD, Paper CThF5. 